\title{
COMPARATIVE EFFICACY OF AMINOQUINOLINE-ANTIFOLATE COMBINATIONS FOR THE TREATMENT OF UNCOMPLICATED FALCIPARUM MALARIA IN KAMPALA, UGANDA
}

\author{
ANNE F. GASASIRA, GRANT DORSEY, BRIDGET NZARUBARA, SARAH G. STAEDKE, ANNETTE NASSALI, \\ PHILIP J. ROSENTHAL, AND MOSES R. KAMYA \\ Makerere University Medical School, Kampala, Uganda; Department of Medicine, San Francisco General Hospital; University of \\ California, San Francisco
}

\begin{abstract}
Resistance to chloroquine (CQ) requires its replacement as first-line therapy for uncomplicated malaria in much of Africa. Combination therapy may improve efficacy and delay the selection of resistant malaria parasites. Combinations of sulfadoxine-pyrimethamine (SP) with 4-aminoquinolines offer affordable and available alternatives to $\mathrm{CQ}$. We conducted a randomized, single-blinded trial to compare the efficacy of SP monotherapy with combinations of SP and either CQ or amodiaquine (AQ) for the treatment of uncomplicated falciparum malaria in patients over 6 months of age in Kampala, Uganda. Of the 448 patients enrolled, 428 (95\%) completed follow-up. Clinical treatment failure after 14 days occurred in 21/140 (15.0\%, 95\% CI 9.5-22.0\%) SP-treated, 11/152 (7.2\%, 95\% CI 3.7-12.6\%) SP/CQ-treated, and 0/136 (0\%, 95\% CI 0-2.7\%) SP/AQ-treated patients. Combination therapies were safe and offered superior efficacy to SP monotherapy. SP/AQ was the most efficacious. This low-cost combination regimen may provide an optimal alternative to CQ for the treatment of uncomplicated malaria in Uganda.
\end{abstract}

\section{INTRODUCTION}

Malaria remains one of the most important infectious diseases worldwide. In sub-Saharan Africa, widespread resistance to chloroquine (CQ), the traditional first-line therapy for uncomplicated malaria, presents an important public health problem. Affected countries are faced with the challenge of selecting a new first-line regimen and revising antimalarial treatment policies. ${ }^{1}$ There is a limited choice of available and affordable antimalarials, and the optimal alternative to $\mathrm{CQ}$ is not clear. Several African countries have adopted sulfadoxine-pyrimethamine (SP) as a replacement for $\mathrm{CQ}{ }^{2}$ However, the therapeutic life span of SP may be limited by rapid emergence of resistance under intense drug pressure. Indeed, evidence for increasing SP resistance in East Africa has begun to emerge. ${ }^{3-6}$

Countries faced with a high prevalence of CQ-resistant malaria are increasingly considering the use of combination therapy to treat uncomplicated malaria. Combination therapy has been advocated to improve efficacy and delay the development and spread of drug resistance. ${ }^{7}$ The use of SP with 4-aminoquinoline antimalarials offers the only available lowcost option for antimalarial combination therapy in Africa at this time. In a systematic review, combinations of SP with either $\mathrm{CQ}$ or amodiaquine (AQ) provided improved fever clearance but similar treatment outcomes to SP monotherapy. ${ }^{8}$ However, resistance to SP was very low in these five studies. With increasing levels of SP resistance, SPaminoquinoline combinations may offer more-significant advantages in therapeutic efficacy.

CQ resistance was first reported in Uganda in $1988,{ }^{9}$ and its prevalence appears to have risen steadily since then. In 2000 , clinical CQ resistance rates of $10-80 \%$ prompted the Ugandan Ministry of Health to re-evaluate its antimalarial treatment recommendations. The combination of SP/CQ was chosen as the provisional replacement for first-line therapy of uncomplicated malaria. Limited information is available regarding the efficacy of $\mathrm{SP} / \mathrm{CQ},{ }^{8,10,11}$ but no trials with this combination have been conducted in Uganda. Recent studies of SP/AQ in Kampala indicated that the combination regimen was safe and highly efficacious for the treatment of uncom- plicated malaria. ${ }^{12,13}$ Importantly, in a longitudinal comparison, SP/AQ was significantly superior to SP and artesunate (AS), an artemisinin derivative widely advocated as a potent new antimalarial. ${ }^{2}$ Both SP/AQ and SP/AS demonstrated excellent efficacy for the treatment of uncomplicated malaria at 14 days of follow-up, but only SP/AS was associated with frequent late-treatment failures. ${ }^{13}$ Thus, in combination with $\mathrm{SP}$, aminoquinolines may offer improved long-term efficacy, in addition to much lower cost, when compared with AS.

To better assess the relative efficacies of aminoquinolineSP combinations, we conducted a randomized trial of SP, $\mathrm{SP} / \mathrm{CQ}$, and SP/AQ for the treatment of uncomplicated malaria in Kampala, Uganda.

\section{MATERIALS AND METHODS}

Subject recruitment. The study was conducted in the outpatient department of Mulago Hospital in Kampala, Uganda from March 2001-January 2002. Malaria is mesoendemic in Kampala, with peak transmission occurring after the two rainy seasons.

Consecutive patients presenting with symptoms suggesting acute malaria and a positive screening blood smear were referred to the study clinic and assessed for eligibility using the following inclusion criteria: 1) age over 6 months; 2) acute uncomplicated Plasmodium falciparum monoinfection with a parasite density of $\geq 2,000$ asexual parasites/ $\mu \mathrm{L}$; 3 ) tympanic temperature of $\geq 38^{\circ} \mathrm{C}$ or history of fever in the previous 48 hrs; 4) absence of concomitant febrile illness; 5) absence of signs and symptoms of severe malaria ${ }^{14}$ or danger signs (convulsion in the previous $24 \mathrm{hrs}$, persistent vomiting, lethargy, inability to drink); 6) absence of known allergy to sulfa drugs; 7) residence in Kampala; 8) provision of informed consent; and 9) agreement to 2 weeks' follow-up.

Recruited patients were interviewed about their symptoms and history of therapy in the previous 2 weeks. A physical examination was performed, and core temperature, height, weight, and spleen size were measured. All temperatures were measured using an electronic tympanic thermometer. A documented fever was defined as a tympanic temperature of $38.0^{\circ} \mathrm{C}$ (equivalent to an axillary temperature of $37.5^{\circ} \mathrm{C}$ ). 
Randomization and treatment. On the day of enrollment, patients were assigned a study number and randomized using a computer generated list to receive either a single dose of SP (Fansidar, Roche), $25 \mathrm{mg} / \mathrm{kg}$ sulfadoxine and $1.25 \mathrm{mg} / \mathrm{kg}$ pyrimethamine; SP plus CQ (Avloclor, Zeneca), $25 \mathrm{mg} / \mathrm{kg}$ in 3 divided doses given on days 0, 1 and 2; or SP plus AQ (Camoquine, Parke-Davis), $25 \mathrm{mg} / \mathrm{kg}$ in 3 divided doses given on days 0,1 and 2. Patients randomized to receive SP were given lactose placebo tablets in the same regimen as CQ. Placebo tablets were not identical to study drugs. All study personnel were blinded to treatment groups except for two nurses who administered treatment but were not involved in outcome assessment. Drugs were administered under direct observation; if a patient vomited within 30 minutes of treatment, the dose was repeated. All patients were given acetaminophen for 3 days to relieve febrile symptoms. Patients with a hemoglobin of $\leq 10 \mathrm{~g} / \mathrm{dL}$ were given ferrous sulfate and mebendazole if they were older than 1 year and had not had an antihelminthic in the previous 6 months, according to Integrated Management of Childhood Illnesses (IMCI) guidelines (Management of Childhood Illnesses, Ministry Of Health, 1997). Patients who failed treatment were given quinine sulfate 10 $\mathrm{mg} / \mathrm{kg}$ every 8 hours for 7 days, and those who developed severe malaria were referred for in-patient care and parenteral quinine.

On day 0 , all recruited patients had phlebotomy done for confirmatory thin and thick blood smears, hemoglobin measurement, and white blood cell (WBC) and neutrophil counts. Alternate patients enrolled had serum alanine aminotransferase (ALT) levels measured. Patients were enrolled into the study if $P$. falciparum monoinfection was confirmed with parasite density of $\geq 2,000$ parasites/ $\mu$ L. Patients were asked to return to the study clinic for follow-up on days 1, 2, 3,7 , and 14 and any other day if they felt unwell.

Follow-up consisted of history-taking, including assessment for adverse events, and a physical examination. A standardized case record form was completed, and if an adverse event was suspected, a separate adverse event form was completed. On follow-up days 3 and 7, and any unscheduled days when the patient had febrile symptoms, a fingerprick was performed for a thick blood smear to assess parasitemia. On day 14, phlebotomy was done to repeat all tests performed on day 0 . Patients who did not return to the clinic on a scheduled follow-up day were visited and assessed at home. Patients whom the home health visitor failed to locate within 24 hours of days 1 to 3 , and 48 hours of days 7 and 14, were classified as lost to follow-up.

Patients were excluded from the study because of: 1) selfmedication with other anti-malarial drugs, 2) development of a concomitant febrile illness that would interfere with the malaria outcome classification, 3) withdrawal of consent, 4) development of danger signs or severe malaria within 24 hours of enrollment.

Thick and thin smears were stained with $2 \%$ Giemsa stain for 30 minutes, and parasite densities and gametocyte counts were calculated based on the number of asexual parasites and gametocytes per $200 \mathrm{WBCs}$, assuming a WBC count of 8,000/ $\mu \mathrm{L}$. Hemoglobin measurement and $\mathrm{WBC}$ and neutrophil counts on days 0 and 14 were measured using a Coulter counter. On day 0 , urine was tested for the presence of 4-aminoquinolines and their metabolites using the Saker-Solomons test. $^{15}$
Outcome measurements. Primary outcome parameters were clinical and parasitologic outcomes at day 14. Parasitologic outcomes were expressed as sensitive (S) or resistant (RI, RII, RIII) according to the World Health Organization (WHO) parasitologic classification system. Clinical outcomes were classified as clinical success (adequate clinical response) or clinical failure (early or late treatment failure) using a slight modification of the recommended WHO clinical classification system. ${ }^{16}$ Patient report of fever in the past 48 hours on days 4-14 in the setting of rising parasitemia was added as a criterion for late treatment failure. Secondary outcomes included: 1) fever (temperature greater than $38^{\circ} \mathrm{C}$ ) clearance; 2) parasite clearance; 3 ) change in hemoglobin from day 0 to day 14; and 4) appearance of gametocytes after day 0.

Tolerability of the study medication also was assessed clinically and by laboratory testing. An adverse event was defined as an unfavorable or harmful symptom or sign or an abnormal laboratory value during or after administration of study medication. For each adverse event, causal relationship with study medication was assessed. Adverse events determined by clinicians to have an unlikely causal relationship with the study medications were not included in the analysis. Adverse event severity was graded as mild, moderate, or severe using the WHO Toxicity Grading Scale and the National Institute of Allergy and Infectious Diseases Division of Microbiology and Infectious Diseases pediatric toxicity tables.

Statistical analysis. Sample size calculations were based on the difference in proportion of parasitologic failure between $\mathrm{SP} / \mathrm{CQ}$ and SP alone. Expected success rates of $70 \%$ in SPtreated and $85 \%$ in SP/CQ-treated patients, respectively, were used. To achieve a one-sided alpha level of $5 \%$ and power of $80 \%$, 136 patients in each group were needed. Assuming a 7\% exclusion rate, our goal was to enroll at least 145 patients in each treatment group.

Data were entered and verified in EPI Info version 6.04 (Centers for Disease Control and Prevention, Atlanta) and analyzed using Stata version 7.0 (Stata, College Station, TX). Proportions were compared using $\chi^{2}$ tests, and continuous variables were compared using Student's $t$ test. Parasite densities were normalized using log-transformation. All patients with known outcomes were included in the analyses. Pairwise comparisons among the treatment groups were planned $a$ priori. Subgroup analysis stratified for ages under 5 and 5 or older were based on previous knowledge of potential effect modification. ${ }^{17}$ Binary outcome data were analyzed by estimating differences in proportions with corresponding exact $95 \%$ confidence intervals $(95 \% \mathrm{CI})$. A $P$ value of $\leq 0.05$ was considered statistically significant. We did not make corrections for multiple comparisons.

Ethical approval. The study was approved by the Faculty Research and Ethical Committee of Makerere University, Kampala, the Uganda National Council for Science and Technology, and the University of California, San Francisco, Committee on Human Research.

\section{RESULTS}

Of 838 patients referred to the study clinic for evaluation, 325 were excluded during the screening interview, primarily because of residence outside Kampala (29\%), unwillingness 


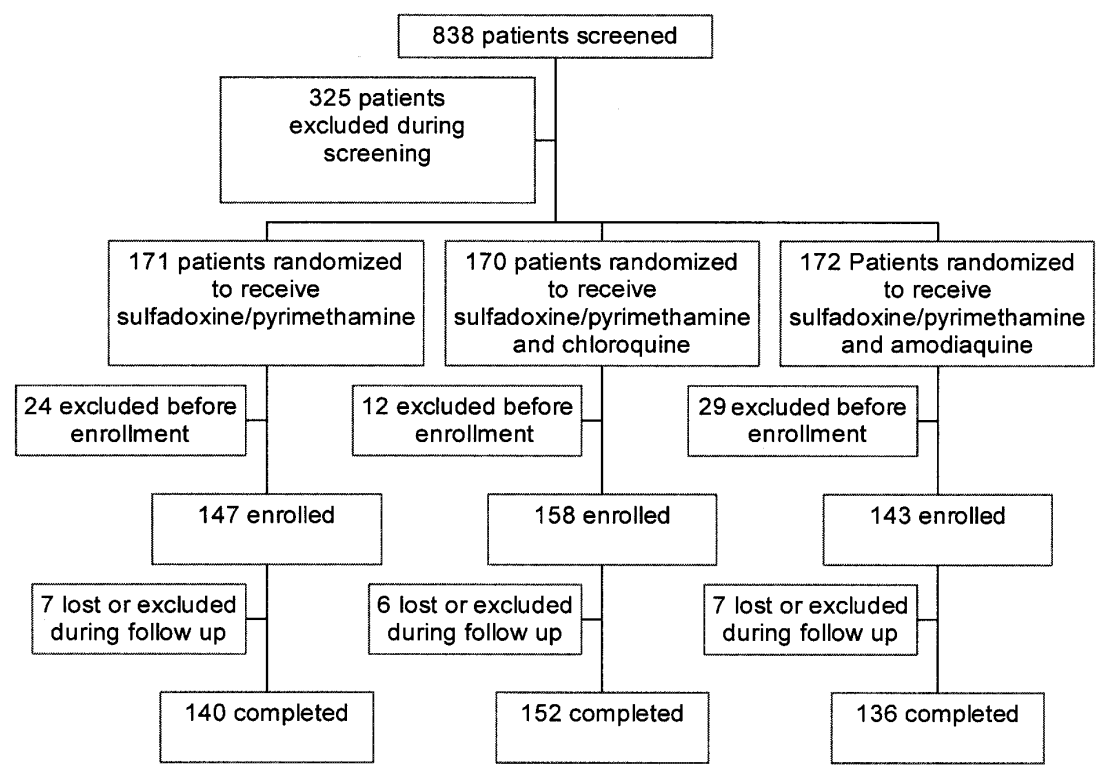

FIGURE 1. Trial profile

to agree to 2 weeks of follow-up (27\%), failure to obtain informed consent $(23 \%)$, and severe disease $(10 \%)$. Of the 513 patients randomized to treatment, an additional 65 were excluded, 35 because of initial parasitemia under $2,000 / \mu \mathrm{L}, 25$ for non-falciparum or mixed infections, and 5 for severe disease within 24 hours of enrollment (Figure 1).

Of the 448 patients enrolled, 428 (95\%) successfully completed the study. Of the 20 patients $(5 \%)$ who did not, nine were treated with additional antimalarials outside the study, five were lost to follow-up, three withdrew informed consent, and three developed a concomitant febrile illness. Patients who did not complete the study were similar to those who completed it in age, initial temperature, and parasite density. (Data not shown.) Among the three treatment groups, patients who completed the study had comparable baseline clinical and demographic characteristics (Table 1).

The risks of clinical and parasitologic failure for the three treatment groups are shown in Table 2. Combination therapies reduced the risk of treatment failure compared with SP monotherapy. SP/AQ significantly reduced the risk of treatment failure compared with both SP monotherapy and SP/
CQ. SP/CQ significantly reduced the risk of treatment failure compared with SP monotherapy. However, when results were stratified by age, the difference in treatment outcome between SP/CQ and SP was significant only in children under the age of 5 (Table 3 ).

Fever resolution differed in the three treatment arms. Patients receiving combination therapy were more likely to be afebrile on day 2 compared with those in the SP monotherapy group (Figure 2). SP/AQ-treated patients were more likely to have no parasites in their blood by day $3(95 \%)$ compared with patients treated with SP/CQ $(84 \%, P=0.005)$ and patients treated with SP alone $(76 \%, P<0.001)$. When comparing SP/CQ to SP alone, there was no significant difference in the proportion of patients with positive smears on day $3(P=$ $0.126)$. Six percent of patients had circulating gametocytes on the day of enrollment, and $18 \%$ developed circulating gametocytes during follow-up. Appearance of gametocytes after commencement of therapy was more common in SP-treated patients $(33 \%)$ compared with patients treated with either SP/CQ $(13 \%, P<0.001)$ or SP/AQ $(7 \%, P<0.0001)$. There was no statistical difference between SP/CQ- and SP/AQ-

TABLE 1

Baseline characteristics of patients who completed the study

\begin{tabular}{|c|c|c|c|}
\hline Characteristics & $\begin{array}{c}\mathrm{SP} \\
\mathrm{n}=140\end{array}$ & $\begin{array}{c}\mathrm{SP} / \mathrm{CQ} \\
\mathrm{n}=152\end{array}$ & $\begin{array}{c}\mathrm{SP} / \mathrm{AQ} \\
\mathrm{n}=136\end{array}$ \\
\hline Female $[\%(95 \% \mathrm{CI})]$ & $56(47-64)$ & $44(36-52)$ & $51(42-59)$ \\
\hline Median age [yrs (range)] & $5.0(0.6-50)$ & $6.0(0.5-44)$ & $6.0(0.5-50)$ \\
\hline Age $<5$ yrs $[\%(95 \% \mathrm{CI})]$ & $47(39-58)$ & $44(36-52)$ & $43(34-51)$ \\
\hline History of antimalarial use in previous 2 weeks $[\%(95 \mathrm{CI})]^{*}$ & $53(44-62)$ & $48(40-56)$ & $61(52-70)$ \\
\hline Temperature $\geq 38.0^{\circ} \mathrm{C}[\%(95 \% \mathrm{CI})]$ & $38(30-46)$ & $39(19-33)$ & $40(31-48)$ \\
\hline Mean hemoglobin $[\mathrm{g} / \mathrm{dL} \pm \mathrm{SD}]$ & $9.7 \pm 2.0$ & $10.2 \pm 2.4$ & $10.2 \pm 2.1$ \\
\hline Hemoglobin $\leq 10 \mathrm{~g} / \mathrm{dL}[\%(95 \% \mathrm{CI})]$ & $55(46-63)$ & $48(40-56)$ & $49(41-58)$ \\
\hline Geometric mean parasite count [parasites $/ \mu \mathrm{L}$ (range)] & 36,700 & 30,620 & 30,410 \\
\hline & $(2,000-710,000)$ & $(2,040-572,000)$ & $(2,000-1,013,760)$ \\
\hline Parasite density $>100,000 / \mu \mathrm{L}[\%(95 \% \mathrm{CI})]$ & $23(16-31)$ & $22(15-29)$ & $21(15-29)$ \\
\hline Positive urine CQ test $[\%(95 \% \mathrm{CI})] \dagger$ & $50(40-60)$ & $55(46-64)$ & $62(51-72)$ \\
\hline
\end{tabular}

$*$ Patients in whom data were recorded

$\dagger$ Patients from whom urine was obtained 
TABLE 2

Clinical and parasitologic treatment outcomes

\begin{tabular}{|c|c|c|c|c|c|c|}
\hline & \multicolumn{2}{|c|}{$\mathrm{SP}(\mathrm{n}=140)$} & \multicolumn{2}{|c|}{$\mathrm{SP} / \mathrm{CQ}(\mathrm{n}=152)$} & \multicolumn{2}{|c|}{$\mathrm{SP} / \mathrm{AQ}(\mathrm{n}=136)$} \\
\hline & $\mathrm{n}(\%)$ & $95 \% \mathrm{CI}$ & $\mathrm{n}(\%)$ & $95 \% \mathrm{CI}$ & $\mathrm{n}(\%)$ & $95 \% \mathrm{CI}$ \\
\hline \multicolumn{7}{|c|}{ Clinical outcomes* } \\
\hline ACR & $119(85 \%)$ & $78-90 \%$ & $141(93 \%)$ & $87-96 \%$ & $136(100 \%)$ & $97-100 \%$ \\
\hline ETF & $7(5 \%)$ & $2-10 \%$ & $3(2 \%)$ & $0-6 \%$ & $0(0 \%)$ & $0-3 \%$ \\
\hline LTF & $14(10 \%)$ & $6-16 \%$ & $8(5 \%)$ & $2-10 \%$ & $0(0 \%)$ & $0-3 \%$ \\
\hline \multicolumn{7}{|c|}{ Parasitologic outcomes* } \\
\hline $\mathrm{S}$ & $99(70 \%)$ & $62-78 \%$ & $126(83 \%)$ & $76-89 \%$ & $135(99 \%)$ & $96-100 \%$ \\
\hline RI & $25(17 \%)$ & $12-25 \%$ & $19(13 \%)$ & $8-19 \%$ & $0(0 \%)$ & $0-3 \%$ \\
\hline RII & $11(8 \%)$ & $4-14 \%$ & $5(3 \%)$ & $1-8 \%$ & $1(1 \%)$ & $0-4 \%$ \\
\hline RIII & $5(4 \%)$ & $1-8 \%$ & $2(1 \%)$ & $0-5 \%$ & $0(0 \%)$ & $0-3 \%$ \\
\hline
\end{tabular}

* $P$-value $<0.001$ for simultaneous comparisons of treatment outcomes among the three treatment groups (Pearson $\chi^{2}$ test)

treated patients in the incidence of gametocytemia after therapy $(P=0.7)$

For all treatment groups, the average hemoglobin levels were higher at the end of follow-up compared with baseline. Fifty percent, $45 \%$, and $48 \%$ of patients randomized to receive $\mathrm{SP}, \mathrm{SP} / \mathrm{CQ}$, and $\mathrm{SP} / \mathrm{AQ}$, respectively, were anemic $(\mathrm{Hb}$ $\leq 10 \mathrm{~g} / \mathrm{dL})$ at baseline. The rate of hematologic recovery in the three groups by day 14 was $36 \%, 46 \%$, and $50 \%$, respectively. The differences in recovery rates were not statistically significant among the three groups $(P=0.3)$

Forty-four percent of the patients who completed the study developed a possible adverse event during therapy or followup. The most frequently reported adverse events are shown in Table 4. Overall, the incidence of possible adverse events was higher in patients receiving combination therapies than in those receiving SP monotherapy. This could be explained by a higher incidence of pruritus and nausea/vomiting in the combination therapies. However, when mild symptoms were eliminated from the analysis, there was no significant difference in frequency of adverse events among the three treatments, and incidence did not exceed $10 \%$ for any symptom reported in any treatment group. In addition, when adverse events considered unlikely to be related to the study medications were included in the analysis, there was no significant difference in number of events among the treatment groups. (Data not shown.) One severe adverse event was reported in an SP-treated patient: an elevated ALT measurement on day 14 of $500 \mathrm{IU} / \mathrm{L}$ (normal range $0-32$ ) in a 3 -year-old girl. On further investigation, other liver enzymes (serum aspartate

TABLE 3

Pairwise comparisons of treatment efficacy

\begin{tabular}{lcc}
\hline & $\begin{array}{c}\text { Clinical outcome } \\
\text { \% risk difference }(95 \% \mathrm{CI})\end{array}$ & $\begin{array}{c}\text { Parasitologic outcome } \\
\text { \% risk difference }(95 \% \mathrm{CI})\end{array}$ \\
\hline All ages & & \\
SP vs. SP/CQ & $7.8(0.6-15.0)$ & $12.2(2.6-21.8)$ \\
SP vs. SP/AQ & $15.0(9.1-20.9)$ & $28.6(20.9-36.2)$ \\
SP/CQ vs. SP/AQ & $7.2(3.1-11.4)$ & $6.4(10.2-22.5)$ \\
Age $<$ 5 years & & \\
SP vs. SP/CQ & $12.2(1.3-23.0)$ & $17.0(2.0-31.9)$ \\
SP vs. SP/AQ & $18.2(8.9-27.5)$ & $36.4(24.8-48.0)$ \\
SP/CQ vs. SP/AQ & $6.0(0.3-11.6)$ & $19.4(10.0-28.9)$ \\
Age $>$ 5 years & & \\
SP vs. SP/CQ & $3.9(-5.6-13.4)$ & $7.7(-4.6-19.9)$ \\
SP vs. SP/AQ & $12.2(4.7-19.6)$ & $21.7(11.8-31.6)$ \\
SP/CQ vs. SP/AQ & $8.2(2.4-14.1)$ & $14.0(6.0-22.1)$ \\
\hline
\end{tabular}

amino-transferase, alkaline phosphatase, and gamma glutamyl transpeptidase) also were elevated. This event was not accompanied by symptoms and resolved without medical intervention within 2 weeks.

\section{DISCUSSION}

In this comparative study, combinations of SP with AQ or CQ were significantly more effective than SP monotherapy for the treatment of uncomplicated malaria in Kampala, Uganda. The SP/AQ combination was significantly superior to the other regimens, providing excellent clinical (100\%) and parasitologic (99\%) efficacy. SP/CQ had intermediate efficacy. Compared with SP monotherapy, treatment with SP/CQ significantly improved clinical and parasitologic outcomes only in children younger than 5 . These results show that combining SP and a 4-aminoquinoline provides more-effective treatment than SP monotherapy. In addition, they highlight the marked superiority in efficacy of SP/AQ over SP/CQ.

Uganda is revising its national antimalarial drug policy, which until recently recommended $\mathrm{CQ}$ for the treatment of uncomplicated malaria. SP/CQ has been adopted as the provisional first-line therapy. Although the SP/CQ combination had not been studied at the time of this policy decision, it was

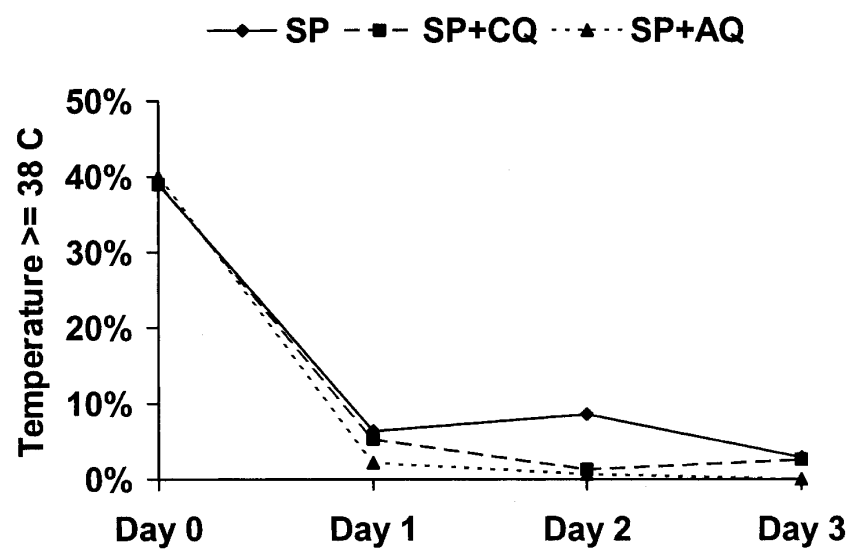

FIGURE 2. Proportion of patients at enrollment and during the first 3 days of follow-up with documented fever. All pairwise comparisons not significant $(P>0.05)$ except combination therapies vs sulfadoxine-pyrimethamine (SP) alone on day 2 (SP/AQ vs SP, $P=0.002, \mathrm{SP} /$ chloroquine vs SP, $P=0.004)$. 
TABLE 4

Proportion of patients with potential adverse events to study medications

\begin{tabular}{lccc}
\hline Adverse event \% (95\% CI) & SP (140) & SP + CQ (152) & SP + AQ (136) \\
\hline Any* & $29(22-38)$ & $49(41-57)$ & $54(45-62)$ \\
Pruritis* & $13(8-20)$ & $34(27-42)$ & $24(17-32)$ \\
Nausea/vomiting $\dagger$ & $10(6-16)$ & $18(12-25)$ & $24(17-32)$ \\
Diarrhea & $7(3-13)$ & $9(5-15)$ & $11(7-19)$ \\
Abdominal pain & $10(6-16)$ & $7(4-13)$ & $6(3-11)$ \\
Neutropenia & $6(2-11)$ & $4(1-8)$ & $9(5-15)$ \\
Cough & $3(1-7)$ & $4(1-8)$ & $5(2-10)$ \\
Anorexia & $4(2-9)$ & $4(1-8)$ & $3(1-7)$ \\
Malaise & $4(2-9)$ & $3(1-8)$ & $3(1-7)$ \\
Headache $\ddagger$ & $6(2-11)$ & $1(0-5)$ & $2(0-6)$ \\
Rash & $2(0-6)$ & $0(0-2)$ & $1(0-4)$ \\
\hline
\end{tabular}

$* P<0.01$ for SP vs. SP $+\mathrm{CQ}$ and $\mathrm{SP}$ vs. $\mathrm{SP}+\mathrm{AQ}$

$\dagger P=0.003$ for $\mathrm{SP}$ vs. $\mathrm{SP}+\mathrm{AQ}$

$\ddagger P=0.01$ for $\mathrm{SP}$ vs. $\mathrm{SP}+\mathrm{CQ}$

All other comparisons $P>0.05$

chosen because of the presumed benefits of combination therapy, lingering reluctance to abandon $\mathrm{CQ}$, and the lack of available data on other antimalarial agents. ${ }^{18}$ This study offers the first comparison in Uganda of the efficacies of SP monotherapy and the SP/CQ combination.

Our study showed benefit when CQ was added to SP, with significant but modest improvement in efficacy based on either clinical or parasitologic criteria. However, considering the fairly high prevalence of SP resistance demonstrated in this and other recent studies, ${ }^{12,13,16}$ and the very high prevalence of $\mathrm{CQ}$ resistance, it seems unlikely that the addition of $\mathrm{CQ}$ to SP will protect it from further selection of resistant parasites. In contrast, as shown in other studies from Kampala, the SP/AQ regimen demonstrated outstanding efficacy against uncomplicated malaria. These data suggest that $\mathrm{SP} /$ $\mathrm{AQ}$, rather than $\mathrm{SP} / \mathrm{CQ}$, is the optimal available combination regimen for the treatment of uncomplicated malaria in Kampala.

For malaria transmission to occur, gametocytes must be transmitted to anopheline mosquitoes. While highly efficacious drugs will reduce gametocytemia because of the rapid reduction of circulating parasites, it has been suggested that treatment with partially effective drugs may increase gametocytogenesis, and this appears to be a particular concern with SP. ${ }^{19-22}$ In this study, the incidence of gametocytemia was significantly higher in SP-treated patients than in either SP/ CQ- or SP/AQ-treated patients, suggesting another advantage of the combination therapies over SP monotherapy.

Attributing adverse events to medications is difficult, as many potential adverse events of antimalarial drugs are similar to signs and symptoms of malaria. Although adverse events were reported more frequently in the combination groups, these differences were due to adverse events of only mild intensity. AQ and SP have both been associated with rare but severe adverse events when used as chemoprophylaxis but appear to be safer when used in short-term therapy. ${ }^{23-26}$ However, in endemic areas where people are treated for malaria repeatedly, the risk of adverse drug reactions may increase. Studies such as this one, which evaluate treatment of a single episode of malaria, are limited in their ability to assess the risk of adverse events with frequent antimalarial use. It is encouraging, therefore, that a longitudinal study in Kampala recently noted no severe adverse events after repeated use of SP/AQ over 1 year. ${ }^{13}$ Careful adverse event monitoring and additional studies evaluating the efficacy, safety, and tolerability of SP/AQ after repeated use will help to more fully assess this highly effective regimen. At present, however, it appears that any potential for increased toxicity from SP/AQ compared with SP/CQ will be offset by markedly improved efficacy, and thus a decreased need for antimalarial treatment over time.

Received July 8, 2002. Accepted for publication October 17, 2002.

Acknowledgments: We thank study team members Denise Njama, Pauline Byakika, Hakim Sendagire, Sr., B.M. Karakire, Sam Nsobya, Moses Kiggundu, Regina Nakafeero, Christopher Bongole, Mary Kasango, Marx Dongo, Assay Balita, Sara Kibirango, and Annette Birabwa, and all patients who participated in the study.

Financial support: UNDP/World Bank/WHO Special Programme for Research and Training in Tropical Diseases (TDR) (RCS/T16/181/ 380 ID No. 970655), the Fogarty International Center/National Institutes of Health (TW00007, TW01506, and AI43301), and the Multilateral Initiative on Malaria.

Authors' addresses: Anne F. Gasasira, Bridget Nzarubara, Annette Nassali and Moses R. Kamya, Makerere University Medical School, P.O. Box 7051, Kampala, Uganda. Grant Dorsey, Sarah G. Staedke and Philip J. Rosenthal, Department of Medicine, P.O. Box 0811, University of California, San Francisco, CA 94143.

Reprint requests: Philip J Rosenthal, Box 0811, University of California, San Francisco, 94143, E-mail: rosnthl@itsa.ucsf.edu

\section{REFERENCES}

1. Bloland PB, Kazembe PN, Oloo AJ, Himonga B, Barat LM, Ruebush TK, 1998. Chloroquine in Africa: critical assessment and recommendations for monitoring and evaluating chloroquine therapy efficacy in sub-Saharan Africa. Trop Med Int Health 3: 543-552.

2. White NJ, Nosten F, Looareesuwan S, Watkins WM, Marsh K, Snow RW, Kokwaro G, Ouma J, Hien TT, Molyneux ME, Taylor TE, Newbold CI, Ruebush TK 2nd, Danis M, Greenwood BM, Anderson RM, Olliaro P, 1999. Averting a malaria disaster. Lancet 353: 1965-1967.

3. Mberu EK, Mosobo MK, Nzila AM, Kokwaro GO, Sibley CH, Watkins WM, 2000. The changing in vitro susceptibility pattern to pyrimethamine/sulfadoxine in Plasmodium falciparum field isolates from Kilifi, Kenya. Am J Trop Med Hyg 62: 396401.

4. Bijl HM, Kager J, Koetsier DW, van der Werf TS, 2000. Chloroquine- and sulfadoxine-pyrimethamine-resistant falciparum malaria in vivo-a pilot study in rural Zambia. Trop Med Int Health 5: 692-695.

5. Ronn AM, Msangeni HA, Mhina J, Wernsdorfer WH, Bygbjerg IC, 1996. High level of resistance of Plasmodium falciparum to sulfadoxine- pyrimethamine in children in Tanzania. Trans $R$ Soc Trop Med Hyg 90: 179-181.

6. Mutabingwa T, Nzila A, Mberu E, Nduati E, Winstanley P, Hills E, Watkins W, 2001. Chlorproguanil-dapsone for treatment of drug-resistant falciparum malaria in Tanzania. Lancet 358: $1218-1223$.

7. White NJ, 1999. Delaying antimalarial drug resistance with combination chemotherapy. Parassitologia 41: 301-308.

8. McIntosh HM, 2001. Chloroquine or amodiaquine combined with sulfadoxine-pyrimethamine for treating uncomplicated malaria. Cochrane Database Syst Rev 4.

9. Sezi CL, Nevil C, Ochen K, Munafu CG, Bekobita D, 1991. The response of Plasmodium falciparum to 4 -aminoquinolines and pyrimethamine/sulfadoxine at six sites scattered throughout Uganda. Uganda Med $J$ 8: 33-46.

10. Bojang KA, Schneider G, Forck S, Obaro SK, Jaffar S, Pinder M, Rowley J, Greenwood BM, 1998. A trial of Fansidar plus chloroquine or Fansidar alone for the treatment of uncomplicated malaria in Gambian children. Trans $R$ Soc Trop Med Hyg 92: $73-76$.

11. Darlow B, Vrbova H, Gibney S, Jolley D, Stace J, Alpers M, 
1982. Sulfadoxine-pyrimethamine for the treatment of acute malaria in children in Papua New Guinea. I. Plasmodium falciparum. Am J Trop Med Hyg 31: 1-9.

12. Staedke SG, Kamya MR, Dorsey G, Gasasira A, Ndeezi G, Charlebois ED, Rosenthal PJ, 2001. Amodiaquine, sulfadoxine/ pyrimethamine, and combination therapy for treatment of uncomplicated falciparum malaria in Kampala, Uganda: a randomised trial. Lancet 358: 368-374.

13. Dorsey G, Njama D, Kamya MR, Cattamanchi A, Kyabayinze D, Staedke SG, Gasasira A, Rosenthal PJ, 2002. Short and longterm efficacies for uncomplicated malaria of sulfadoxine/ pyrimethamine alone or in combination with amodiaquine or artesunate: a longitudinal randomized trial in Ugandan children. Lancet (in press).

14. World Health Organization, 1990. Severe and complicated malaria. Trans $R$ Soc Trop Med Hyg 84: 1-65.

15. Mount D, Nahlen B, Patchen L, Churchill F, 1989. Adaptations of Saker-Solomons test: simple, reliable colorimetric field assays for chloroquine and its metabolites in urine. Bull WHO 67: 295-300.

16. World Health Organization, 1996. Assessment of therapuetic efficacy of antimalarial drugs for uncomplicated malaria in areas with intense transmission. Geneva: WHO.

17. Kamya MR, Dorsey G, Gasasira A, Ndeezi G, Babirye JN, Staedke SG, Rosenthal PJ, 2001. The comparative efficacy of chloroquine and sulfadoxine-pyrimethamine for the treatment of uncomplicated falciparum malaria in Kampala, Uganda. Trans R Soc Trop Med Hyg 95: 50-55.

18. Kamya MR, Bakyaita NN, Talisuna AO, Were WM, Staedke SG, Increasing antimalarial drug resistance in Uganda and revision of the national drug policy. Trop Med Int Health (in press).
19. von Seidlein L, Jawara M, Coleman R, Doherty T, Walraven G, Targett G, 2001. Parasitaemia and gametocytaemia after treatment with chloroquine, pyrimethamine/sulfadoxine, and pyrimethamine/sulfadoxine combined with artesunate in young Gambians with uncomplicated malaria. Trop Med Int Health 6: 92-98.

20. Robert V, Awono-Ambene HP, Le Hesran JY, Trape JF, 2000. Gametocytemia and infectivity to mosquitoes of patients with uncomplicated Plasmodium falciparum malaria attacks treated with chloroquine or sulfadoxine plus pyrimethamine. Am J Trop Med Hyg 62: 210-216.

21. Sokhna CS, Trape JF, Robert V, 2001. Gametocytaemia in Senegalese children with uncomplicated falciparum malaria treated with chloroquine, amodiaquine or sulfadoxine + pyrimethamine. Parasite 8: 243-250.

22. Price R, Nosten F, Simpson JA, Luxemburger C, Phaipun L, ter Kuile F, van Vugt M, Chongsuphajaisiddhi T, White NJ, 1999. Risk factors for gametocyte carriage in uncomplicated falciparum malaria. Am J Trop Med Hyg 60: 1019-1023.

23. Felix H, Danis M, 1987. [Malaria prevention today and tomorrow.] Bull Soc Pathol Exot Filiales 80: 581-592.

24. Nevill CG, Verhoeff FH, Munafu CG, ten Hove WR, van der Kaay HJ, Were JB, 1994. A comparison of amodiaquine and chloroquine in the treatment therapy of falciparum malaria in Kenya. East Afr Med J 71: 167-170.

25. Phillips-Howard PA, West LJ, 1990. Serious adverse drug reactions to pyrimethamine-sulphadoxine, pyrimethaminedapsone and to amodiaquine in Britain. $J$ R Soc Med 83: 82-85.

26. Olliaro P, Nevill C, LeBras J, Ringwald P, Mussano P, Garner P, Brasseur P, 1996. Systematic review of amodiaquine treatment in uncomplicated malaria. Lancet 348: 1196-1201. 